\title{
$\widehat{A}$ Madridge \\ madridge Journal of Immunology \\ Interconnecting Scientific World
}

Review Article

Open Access

\section{The Mechanism of HIV-1 Latency and Therapeutic Implication}

\author{
Adekunle Sanyaolu ${ }^{1 *}$, Danil Hammoudi ${ }^{2}$, Olanrewaju Badaru ${ }^{1}$, Ifeoluwa Adekanye ${ }^{3}$, Adeola Akinwekomi ${ }^{3}$ and \\ Kateryna Shyshkova ${ }^{3}$ \\ ${ }^{1}$ Federal Ministry of Health, Abuja, Nigeria \\ ${ }^{2}$ Sinoe Medical Association, Baltimore, Maryland, USA \\ ${ }^{3}$ Saint James School of Medicine, Anguilla
}

\section{Article Info}

\section{*Corresponding author:}

Adekunle Sanyaolu

Professor

Federal Ministry of Health

Abuja, Nigeria

Nigeria

E-mail: sanyakunle@hotmail.com

Received: February 20, 2019

Accepted: February 22, 2019

Published: February 28, 2019

Citation: Sanyaolu A, Hammoudi D, Badaru O, Adekanye I, Akinwekomi A, Shyshkova K. The Mechanism of HIV-1 Latency and Therapeutic Implication. Madridge J Immunol. 2019; 3(1): 78-83

doi: $10.18689 / \mathrm{mjim}-1000118$

Copyright: ( $) 2019$ The Author(s). This work is licensed under a Creative Commons Attribution 4.0 International License, which permits unrestricted use, distribution, and reproduction in any medium, provided the original work is properly cited.

Published by Madridge Publishers

\begin{abstract}
The latency of resting HIV-1 in CD4+ T cells is the obstacle to complete destruction of the virus in patients that have been given highly active antiretroviral therapy (HAART). This latency occurs early during acute infection but remains silent in the host cells; however it is still capable of making infectious proviruses if antiviral therapy is stopped. The goal of HAART therapy is to reduce the replication levels of HIV-1 to undetectable levels in serum of infected individuals. HIV-1 therapy involves the use of multiple drugs because of the ability of the virus to easily acquire drug resistance to an inhibitor. Resistance develops due to the diversity of HIV-1 genome within several individuals. Gene therapy approaches have been shown to be somewhat safer but have not been proven yet in human models. The process of HIV-1 latency is multifactorial and involves several molecular pathways which are still being studied. This study reviews HIV-1 latency as well as its therapeutic implication.
\end{abstract}

Keywords: HIV-1; Latency; HAART; CD4+ T cells.

\section{Introduction}

Human immunodeficiency virus type 1 causes symptoms of acquired immunodeficiency syndrome, otherwise known as AIDS. Type 1 is the most common type of HIV infection; a great percentage of the world is mostly affected with this form of HIV. HIV-2 has a longer latent period between time of infection and effect as compared with HIV-1. Structurally, both types have similar enzymes but differ in their genes. HIV2 is more common in West Africa [1]. Immunologically, HIV involves the destruction of CD4+ lymphocytes, which are important for cell mediated immunity in healthy individuals. When these lymphocytes are destroyed, it reduces its number in the body thereby making individuals more susceptible to various opportunistic infections. Once the CD4+ count is less than 200 per microliter, individuals are likely to develop AIDS [2].

HIV can result in the impairment of several organs in the body and even worsen the survival of current diseases. One of the main problems in treating HIV is the latency period associated with it. Treatment is aimed at reducing the replication by using several drugs that could inhibit the HIV enzymes. Currently, over 30 non-identical anti-HIV drugs have been accepted for use in the clinical setting to target varying steps in the viral life cycle [3]. The latency of resting HIV-1 in CD4+ T cells is the obstacle to complete destruction of the virus in patients that have been given highly active antiretroviral therapy (HAART). This latency occurs early during acute infection but remains silent in the host cells; however it is still capable of making infectious proviruses if antiviral therapy is stopped $[4,5]$. 
HIV is a T-cell lympho-tropic retrovirus that belongs to the T-cell leukemia virus (HTLV) group. The virus is composed of two positive-senses single-stranded RNA which are not linked covalently, with the viral gene encoded in each of these RNA copies. The genome consists of the following genes; gag, rev, pol, vpr, vpu, vif, tat, nef, and LTR. These genes are also in HIV2 with the exception of the vpu gene. In HIV-2, the vpu gene is replaced with a unique vpx gene that encodes a $16-\mathrm{kDa}$ protein $[6,7]$. Viral protein $U(\mathrm{vpu})$ enhances the budding of the virus particle from the host cell, with a 10 to $20 \%$ increase in production. In addition, it enhances the destruction of CD4 proteins, thereby decreasing super infection and elongating the life of the host cell a little longer. Research has shown that "viral protein $x^{\prime}$ might have a relation to vpr which increases the production of HIV proteins, but this has not been fully proven. Moreover, the vpx gene has been seen to not have much effect in HIV-2 because without the gene the virus continues to function, replicate, and infect cells $[8,9]$.

\section{HIV-1 Life Cycle}

The cycle starts with binding of the viral envelope protein to receptor target cells of CD4 and CCR5. This binding allows for fusion of the viral gp120 with the outer membrane of host cell; as a result, viral particles enter the cell. Next, reverse transcriptase forms one double-stranded viral DNA molecule; it does this by replicating the RNA sequence in the virus. The DNA of the virus becomes integrated in the nucleus of the host DNA, which is then referred to as a provirus. The provirus works as a base to form new viral RNA, after transcription, the new viral RNA exit the nucleus of cells that were infected. The new viral RNAs are translated into polypeptide chains that eventually become the proteins and enzymes of the new virus particles. Furthermore, the newly formed proteins, enzymes, etc. are assembled on the outer membrane to prepare for release. Lastly, the accumulated buds that were assembled are cut from the membrane, allowing the release of the virus particles. During the process of assembly, protease enzyme breaks the polypeptide chains into many positions with a purpose to make the viral particles more infectious. Once particles are mature and released, it is capable of infecting new host cells, thus repeating the cycle [10].

\section{Antiretroviral Therapy for HIV-1 Infection}

Highly active antiretroviral therapy aims to elongate survival and decrease mortality rates of individuals infected with the HIV virus. HAART consists of a combination of several medications that are used in patients depending on viral load and CD4+ cell count. The goal of this therapy is to reduce the replication levels of HIV-1 to levels that should not be detected in serum of infected individuals. There are six classes drugs that are used to target enzymes at different stage of HIV-1 life cycle; nucleoside reverse transcriptase inhibitors (NRTIs), non-nucleoside reverse transcriptase inhibitors (NNRTIs), protease inhibitors (PIs), fusion inhibitors (Fls), chemokine receptor antagonists and integrase strandtransfer inhibitors (INSTIs) [11].
The first group of drug to be approved was NRTIs, and they form the backbone of HIV treatment because of the favorable pharmacokinetic profile. These drugs are nucleoside analogs, which include Zidovudine, Abacavir, Didanosine, Emtricitabine, Lamivudine, Stavudine, Tenofovir DF, Tenofovir $A F$, and Zalcitabine [12]. The mechanism of action involves interruption of HIV replication by inhibiting reverse transcriptase competitively; hence, terminating viral DNA chain. NNTRIs are as well integral part of initial treatment option in HIV-1 infection. They differ from NTRIs in that they do not require phosphorylation to become active, but have similar mechanism of action. Drugs in this category include nevirapine, efavirenz, delaviridine, etravirine, and rilpivirine [12]. These drugs have activity against HIV-1.

Pls prevent cleavage of polypeptide precursors into functional subunits and inhibit the assembly of new viruses by competitively binding to HIV protease [13]. Although the drugs in this category have similar mechanism of action, they differ slightly in efficacy and pharmacokinetics. Integrase inhibitors prevent the transport of proviral DNA and attachment to host chromosomes. Low frequency of side effects is observed with this group of drugs because humans do not have homologue for HIV integrase [13]. Fusion inhibitors prevent the exposure of the gp41 terminal domain thus preventing insertion through the fusion peptide. Chemokine receptor antagonists bind to either CCR5 or CXCR4 coreceptors, thereby blocking viral entry [14].

\section{Resistance}

HIV-1 treatment involves the use of multiple drugs because of the ability of the virus to easily acquire drug resistance to an inhibitor. Resistance is due to the diversity of HIV-1 genome within several individuals, this resistance results in an increased replication of the virus with nature of RT and recombination being error-prone. Though the continuous use of HAART has shown significant improvement in the treatment of HIV-1, the resistance of the virus to several drugs is still emerging; thus creating a major issue with treatment. Resistance of the virus does not only pertain to the use of multiple drugs, it can also be due to drug toxicity in relation to a poor adherence by the patient. Also, the transmission of new infections can lead to failure of multiple drugs [15].

Identifying resistance to drug involves the use of a 3-step process, nucleic acid analysis of HIV genome, determining mutations related to drug resistance and creating a drug resistant report [16]. Though there is no cure for HIV yet, researchers are looking for ways to better understand the mechanisms of HIV-1 replication, thus being able to discover better therapeutic treatment.

\section{Cellular Reservoirs/Nature of Cellular Reservoirs}

As defined by Van Lint, Bouchat, \& Marcello, a viral reservoir is an anatomical site or a cell type where the virus remains for a longer duration of time and is competent to 
replicate [17]. Reservoirs for HIV are defined as the infected cell that house dormant virus or where replication occurs at low levels. According to Le Douce, Herbein, Rohr, \& Schwartz, the major cellular reservoir found in natural host are memory CD4+ T cells, especially subsets of resting central memory $\mathrm{T}$ cells (TCM), and translational memory $\mathrm{T}$ cells (TTM) [18]. Although the frequency of infection in naïve $\mathrm{T}$ cells is less compared to memory $\mathrm{T}$ cells, these cells have been demonstrated to house latent proviruses as well [19].

Several studies on detailed analysis of residual viremia have shown that there are additional reservoirs. Tissue macrophages which are also major targets in patients infected with HIV-1 are possible reservoir for the virus because of the presence of both CD4 receptors and Coreceptors CXCR4 or CCR5 on their surface [19]. It is important to note that HIV-1 viruses in these cells are not completely silent, but replicate at a lower rate. In contrast to T-cells, macrophages extend the lifespan of the virus and make them more resistant to apoptosis. HIV-1 has no cytopathic effect on cells from the lineage of monocyte/ macrophage; as a result, these cells can house the virus longer than T cells [17]. Also, most of the organ systems of the body have macrophages, which make these cells capable of distributing the virus throughout the body. The major macrophages that have been known to harbor HIV-1 are the central nervous system (CNS) resident macrophages, and dendritic cells [19].

Resident macrophages of CNS are meningeal macrophages, perivascular macrophages, microglia, and macrophages of choroid plexus; these cells serve as reservoir for HIV-1 virus. Microglial cells and perivascular macrophages are significantly important clinically because infection of these cells is associated with dementia seen in HIV-1 patients [19]. The turnover rate of these two cells is low with perivascular macrophages having about 2-3 months turnover rate, and microglial cells several years; this makes them important CNS reservoirs for HIV-1. The bridge between innate and adaptive immune system is dendritic cell. All subtypes of this cell, plasmacytoid dendritic cells, langerhans cells, and myeloid cells, are prone to HIV-1 infection.

Another cell that has been speculated to contain latent provirus is hematopoietic progenitor cells (HPC). This conclusion was made from the observation that HIV-1 patients have hematopoietic abnormalities [19]. Studies have shown that the virus infects multi potent HPCs, and latency is established in these cells.

\section{HIV-1 Latency}

The process of HIV-1 latency is multifactorial and involves several molecular pathways; although several studies are still being conducted to better understand the importance of each pathways involved [20]. Latency is a reversible state of infection where production of viral nucleic acid is low, but infected cells still possess the ability to produce new viral particles. However, target cells must be activated in order for productive infection to occur [21].
During HIV-1 infection, there's integration of viral genetic material into host cells where they replicate. These infected cells often produce infectious proviral DNA until they are killed off by host immune system, majorly by $T$ cells or the virus itself. Also, several replicative-defective proviruses are incorporated into the host genome as well $[22,23]$. This integration allows for the persistence of HIV-1 genome within infected hosts. The biological processes that drive the virus into latency and control repression of transcription are broadly classified into two based on chromosomal integration; pre-integration latency and postintegration latency [22,23].

\section{Pre-Integration latency}

The prerequisite for favorable HIV-1 pathogenesis is incorporation of viral DNA into host genome. Upon cellular entry, RNA of the virus is transcribed into DNA via reverse transcriptase. The DNA is assembled as pre-integration complex (PIC) which compose of dsDNA and proteins; Vpr, matrix, integrase and capsid [19]. It is the PIC that is transported and incorporated into the nucleus of host cell where it is taken into host genome. Efficacy problems during the process of reverse transcription or problems with PIC nuclear transport can give rise to pre-integration latency. This form of latency is often seen in CD4+ T cells and controlled majorly by interactions with host restriction factors [19]. However, this only contributes a small fraction to viral latency because $T$ cells have very short half-lives of about a day [13].

This form of latency is also observed in cells from the lineage of monocyte/macrophage, and this has a major contribution to both the persistence and dissemination of HIV-1 in infected hosts. Vpr, which is an accessory HIV-1 protein is important for viral replication in these cells [18]. Hence, the deletion of this protein result in up to 10 times decrease in viral transcription. Also, impaired nuclear transport of PIC resulting from low level of ATP pool, and low level of dNTP can contribute to inefficient transcription of the provirus.

\section{Post-integration latency}

As the name suggests, this form of latency occurs after the completion of integration into host genome. Several molecular mechanisms play important roles in silencing gene expression of the virus. According to Kumar et al. these mechanisms include transcription gene silencing (TGS), epigenetic gene silencing, and post-transcriptional gene silencing [19]. The persistence and spread of HIV-1 is majorly a result of post-integration latency. Latency of this form has been described in both T-cells and cells of monocyte/ macrophage lineages. For CD4+ cells, post-integration latency is mostly controlled by chromatin environment of infected host [18]. In most cases, latency is established when viral genome is integrated into repressive heterochromatin. Although integration into active euchromatin can also be responsible for establishment of viral latency especially when there is transcriptional interference [18]. 
HIV-1 Tat protein, a potent transactivator, has also been described to be involved in the pathogenesis of post-integration latency in infected cells. The lack of Tat transactivation results in the restriction of HIV-1 transcription [18]. Activation of Tat involves recruitment of positive transcription elongation factor (pTEFb), which composes of proteins cyclin dependent kinase 9 (Cdk9) and cyclin T1 (CycT1). Expression of CycT1 is a limiting factor for PTEFb function; hence, low cellular level of this protein could result in lack of transactivation of Tat. In addition, phosphorylation of CDK9 is essential for its normal function in the differentiation of monocytes into macrophages; hence, low levels of cellular ATP or conditions that prevent phosphorylation of the protein could also result in inadequate transactivation. Post-integration latency in monocyte/macrophage lineage could result from lack or loss of function of tat protein, the presence of transcriptional repressors, chromatin environment influence, lack of transcriptional activators, and mi RNA [18].

\section{Genetic Markers}

There are several cellular factors that influence the latency of HIV-1 in infected host. Cellular transcription factors, Tat, and miRNA have all been studied to contribute to this phenomenon [20]. Proviral DNA of HIV-1 is enclosed by long terminal repeats (LTR) at both $5^{\prime}$ and $3^{\prime}$ ends. The $5^{\prime}$ LTR acts as a transcriptional regulator because of the presence of binding sites for several transcription factors such as C-Myc, NF-k $\beta$, USF, AP1, COUP, TCF- $1 \alpha$, SP1 $[19,24]$. These factors act together to control the transcription of HIV-1 provirus. SP1, for example, recruits C-Myc to the $5^{\prime}$ LTR. This association result in further recruitment of histone deacetylation 1 (HDAC1), which prompts remodeling of chromatin responsible for suppression of viral gene expression. Other factors that recruit HDACs to the promoter region of the provirus, hence causing latency include COUPTF interacting protein 2 (CTIP2), LSF, C-promoter binding factor-1 (CBF-1), and YY1 [19,24].

\section{Reactivation of Latent HIV-1 Proviruses}

\section{Initiation of transcription}

There is a wide range of factors that can induce the activation of proviruses ranging from cytokines, TNF- $\alpha$, and mitogens among others. These factors activate T-cell and cause an effect in the gene expression of both host cell and virus via signaling pathways. This signaling results in the activation of cellular transcription factors, and ultimately leads to HIV-1 transcription [25]. Several DNA binding domains are present in the viral LTR which allows for interaction of various factors. Of these factors, the important ones required for HIV-1 replication are SP1 and NF-kB.

Depending on the member of Rel family that is bound to $N F-k B$, it can serve as both transcriptional activator and repressor [25]. Binding of NF-kB p50 homodimer to HIV LTR promote recruitment of repressors which inhibits transcription of provirus. Activation of T cells results in displacement of p50 homodimer by $\mathrm{p} 50$-RelA heterodimer which result in histone acetylation; hence promoting transcriptional initiation. Studies have shown that low levels of p50-RelA heterodimers cause a reduced level of HIV-1 transcription. A member of the Rel family, c-Rel serves as a competitive inhibitor of RelA binding site on the LTR; hence, inhibits activation of HIV transcription [25].

HIV-1 gene expression can also be activated by induction of AP-1 and NFAT by ligation of T-cell receptor. NFAT has been shown to be an important factor in the reactivation of latency especially in memory $T$ cells, and function via IL-7 dependent pathway [25]. HIV transcription is also influenced by non-inducible transcription factors; LEF/ TCF1-a, USFA and Ets promote expression of HIV-1 provirus, while HIV-1 transcription is repressed by the binding of YY1 factor [25].

\section{Stimulation of RNA polymerase II elongation}

Upon initiation of transcription, several cellular proteins are required to promote efficient expression of HIV-1 transcript. HIV-1 transactivating protein Tat plays a major role to ensure effective elongation by Pol II. Tat binds to the $5^{\prime}$ region of the RNA transcript and induces the recruitment of various cellular factors that promote efficiency of Pol II. Transcriptional activity of Tat depends on its interaction with P-TEFb (a cellular kinase comprised of CDK9 and CyclinT1 heterodimers). Binding of this protein to RNA Pol II phosphorylates it and results in effective elongation process. Fully spliced viral transcripts are exported out host nucleus once transcription is complete. For Tor effective export, viral protein $\operatorname{Rev}$ is required $[26,27]$.

\section{Pharmacological Approach to Eradicate Latency}

Most approaches to HIV-1 infections involves the use of HAART to halt the replication of the virus thereby reducing its clinical appearance, however, the problem with HAART is its inability to target the latency within the genome of the virus. This specific issue is the basis of therapeutic and pharmacological approaches. Where ARTs fail to penetrate sections, such as the CNS and gut-associated lymphoid tissues, pharmacological approaches might succeed. Most current pharmacological strategies involve the reactivation of HIV expression within reservoir cells enabling vulnerability to eliminate host immune response and viral cytopathic effects [28].

The use of HDAC inhibitors (HDAC is) have been shown to serve as an advantage in the pharmacology of HIV-1 latency. Because HDACis have been used for several years for other medical issues including cancer, HDACis are being improved for better use in HIV patients. This is mainly due to its lipophilic nature thus having the ability to target certain sections of the body like the CNS. Moreover, the use of VPA valproic acid in combination with ART was shown to decline reservoir of HIV infected patients in 3 out of 4 patients. Without combining ART, the efficiency of elimination of the reservoirs was not as effective. Prostratin has also been 
shown to be effective in activating the expression of HIV virus from latent infected cells. Combining HDACi with prostratin has been shown to work hand in hand by down regulating the expression of HIV receptors thus being able to prevent the infection.

The use of 5-hydroxynaphthalene-1,4-dione (HN), has been shown to induce latent infection without activating $T$ cell. Though studies have shown that this compound might not be effective for clinical studies, researchers have proven that compounds that activate NF-KB but do not activate $T$ cells will have a better chance at eliminating the reservoirs of the infection [28].

Other techniques aimed at reversing the latent state include the use of chromatin reshaping components such as $T$ cell activators and differentiating agents, and acetylation and methylation inhibitors. [29]. Recently, the use of suberoylanilide hydroxamic acid SAHA that is an HDAC inhibitor has been approved for use in clinical studies. Its market name vorinostat is used to treat cutaneous leukemia and has also shown to activate latent infected cells of HIV-1. The drug has been shown to disrupt latent infection within the reservoir of the resting CD4 positive cells. It aims at acetylating histone 3 and transcribing HIV RNA in these resting cells [29].

Another therapeutic approach involves immune based therapies that aim at reactivation of compounds to improve the removal of latent infected cells that have become reactivated with an effort to enhance response to immunity. Recently, the use of cytokines with interleukins 2, 7, and 15 in combination with ART is being tested to reverse the negative regulators of immune activation. However, some of the tests have been shown to yield adverse side effects [29]. Furthermore, gene therapy approaches have been shown to be somewhat safer but have not been proven in human models yet [29]. There is continuous ongoing research aimed solely to eradicate the reservoirs of the virus but so far clinical trials and models haven't been 100\% successful.

\section{Conclusion}

Currently, the treatment of HIV-1 infection involves the use of HAART, but this method does not completely eradicate the virus from infected individuals. Recent studies have identified the long-lived reservoirs of HIV-1 which contribute to the persistence of the virus in patients, despite treatment with antiretroviral therapy. As a result of the presence of latency, there has been long-term toxicity observed in patients on HAART. It is increasingly clear now that HIV-1 latency is regulated by both viral and cellular factors. It is essential to determine the various factors that reinforce or antagonize latency, such as the role of chromatin in viral latency, and the cellular natures of viral reservoirs as well as agents that are capable of effectively activating virus from latent reservoirs. Hence, before complete viral eradication can be achieved, these reservoirs must be eliminated through therapeutic approach.
A major goal in the field of HIV research is to identify a safe and effective cure for the millions of people living with HIV around the world. A cost-effective strategy for limiting the spread of the virus and eliminating lifelong therapy can be achieved when infected individuals can be permanently cured. It is only through deeper knowledge that would make it possible to discover effective combination therapies that could completely eradicate latent reservoirs in infected patients; hence, leading to complete cure for HIV-1 infected patients.

\section{References}

1. Nyamweya $S$, Hegedus $A$, Jaye $A$, Rowland-Jones $S$, Flanagan $K L$, Macallan DC. Comparing HIV-1 and HIV-2 infection: Lessons for viral immunopathogenesis. Rev Med Virol. 2013; 23(4): 221-240. doi: 10.1002/ rmv.1739

2. McMichael AJ, Borrow P, Tomaras GD, Goonetilleke N, Haynes BF. The immune response during acute HIV-1 infection: clues for vaccine development. Nat Rev Immunol. 2010; 10(1): 11-23. doi: 10.1038/nri2674

3. Ruelas DS, Greene WC. An Integrated Overview of HIV-1 Latency. Cell. 2013; 155(3): 519-529. doi: 10.1016/j.cell.2013.09.044

4. Bosque A, Planelles V. Induction of HIV-1 latency and reactivation in primary memory CD4+ T cells. Blood. 2009; 113(1): 58-65. doi: 10.1182/ blood-2008-07-168393

5. Contreras $X$, Lenasi T, Peterlin BM. HIV latency: present knowledge, future directions. Future Virol. 2006; 1(6): 733-745. doi: 10.2217/17460794.1.6.733

6. Lin LI, Li HS, Pauza CD, Bukrinsky M, Richard YZ. Roles of HIV-1 auxiliary proteins in viral pathogenesis and host-pathogen interactions. Cell Res. 2005; 15(11): 923-34. doi: 10.1038/sj.cr.7290370

7. Marcello A. Latency: the hidden HIV-1 challenge. Retrovirology. 2006; 3: 7. doi: 10.1186/1742-4690-3-7

8. Anderson I, Low JS, Weston S, et al. Heat shock protein 90 controls HIV1 reactivation from latency. Proc Natl Acad Sci USA. 2014; 111(15): 15281537. doi: $10.1073 /$ pnas. 1320178111

9. Das AT, Harwig A, Berkhout B. The HIV-1 Tat Protein has a Versatile role in activating Viral Transcription. J Virol. 2011; 85(18): 9506-9516. doi: 10.1128/JVI.00650-11

10. Campbell EM, Hope TJ. Live cell imaging of the HIV-1 life cycle. Trends Microbiol. 2008; 16(12): 580-587. doi: 10.1016/j.tim.2008.09.006

11. Desai M, lyer G, Dikshit RK. Antiretroviral drugs: Critical issues and recent advances. Indian J Pharmacol. 2012; 44(3): 288-298. doi: 10.4103/02537613.96296

12. Abbas W, Herbein G. Molecular Understanding of HIV-1 Latency. Adv Virol. 2012; 574967. doi: 10.1155/2012/574967

13. Rathbun $C$, Liedtke MD. Antiretroviral Therapy for HIV Infection. Medscape 2016.

14. Adamson CS, Freed EO. Novel Approaches to Inhibiting HIV-1 Replication. Antiviral Res. 2010; 85(1): 119. doi: 10.1016/j.antiviral.2009.09.009

15. Laird GM, Bullen CK, Rosenbloom DI, et al. Ex vivo analysis identifies effective HIV-1 latency-reversing drug combinations. J Clin Invest. 2015; 125(5): 1901-1912. doi: 10.1172/JCl80142

16. Cortez KJ, Maldarelli F. Clinical Management of HIV Drug Resistance. Viruses. 2011; 3(4): 347-378. doi: 10.3390/v3040347

17. Van Lint C, Bouchat S, Marcello A. HIV-1 transcription and latency: an update. Retrovirology. 2013; 10: 67. doi: 10.1186/1742-4690-10-67

18. Le Douce V, Herbein G, Rohr O, Schwartz C. Molecular mechanisms of HIV-1 persistence in the monocyte-macrophage lineage. Retrovirology. 2010; 7: 32. doi: 10.1186/1742-4690-7-32

19. Kumar A, Abbas W, Herbein G. HIV-1 latency in monocytes/macrophages. 
Viruses. 2014; 6(4): 1837-60. doi: 10.3390/v6041837

20. Donahue DA, Wainberg MA. Cellular and molecular mechanisms involved in the establishment of HIV-1 latency. Retrovirology. 2013; 10: 11. doi: $10.1186 / 1742-4690-10-11$

21. Tolstrup M. Editorial commentary: Reversing latency in HIV-infected patients. Clin Infect Dis. 2014; 58(6): 891-892. doi: 10.1093/cid/cit814

22. Taube R, Peterlin BM. Lost in Transcription: Molecular Mechanisms that Control HIV Latency. Viruses. 2013; 5(3): 902-927. doi: 10.3390/v5030902

23. Han $Y$, Wind-Rotolo $M$, Yang HC, Siliciano JD, Siliciano RF. Experimental approaches to the study of HIV-1 latency. Nat Rev Microbiol. 2007; 5(2): 95-106. doi: 10.1038/nrmicro1580

24. Kim HG, Kim KC, Roh TY. Gene silencing in HIV-1 latency by polycomb repressive group. Virol J. 2011; 8: 179. doi: 10.1186/1743-422X-8-179

25. Williams SA, Greene WC. Regulation of HIV-1 Latency by T-cell
Activation. Cytokine. 2007; 39(1): 63-74. doi: 10.1016/j.cyto.2007.05.017

26. Kauder SE, Bosque A, Lindqvist A, Planelles V, Verdin E. Epigenetic Regulation of HIV-1 Latency by Cytosine Methylation. PLoS Pathog. 2009; 5(6): e1000495. doi: 10.1371/journal.ppat.1000495

27. Tyagi M, Pearson RJ, Karn J. Establishment of HIV Latency in Primary CD4 cells is due to Epigenetic Transcriptional Silencing and P-TEFb Restriction. J Virol. 2010; 84(13): 6425-6437. doi: 10.1128/JVI.01519-09

28. Choudhary SK, Margolis DM. Curing HIV: Pharmacologic approaches to target HIV-1 latency. Annu Rev Pharmacol Toxicol. 2011; 51: 397-418. doi: 10.1146/annurev-pharmtox-010510-100237

29. Battistini A, Sgarbanti M. HIV-1 Latency: An Update of Molecular Mechanisms and Therapeutic Strategies. Viruses. 2014; 6(4): 1715-1758. doi: $10.3390 / v 6041715$ 